\title{
The value of the apparent diffusion coefficient value in the Liver Imaging Reporting and Data System (LI-RADS) version 2018
}

\author{
Gehad Ahmad Saleh ${ }^{1, B, C, D, E}$, Ahmed Abdel Khalek Abdel Razek, ${ }^{1, A, E, F}$, Lamiaa Galal El-Serougy, \\ Walaa Shabana ${ }^{2, C, D, E}$, Rihame Abd El-Wahab ${ }^{1, B, C, D, E, F}$ \\ 'Department of Diagnostic Radiology, Mansoura University, Faculty of Medicine, Mansoura, Egypt \\ 2Department of Tropical Medicine, Mansoura University, Faculty of Medicine, Mansoura, Egypt
}

\section{Abstract}

Purpose: To assess role of the apparent diffusion coefficient (ADC) in the Liver Imaging Reporting and Data System (LI-RADS) version 2018 for the prediction of hepatocellular carcinoma (HCC).

Material and methods: Retrospective analysis of 137 hepatic focal lesions in 108 patients at risk of HCC, who underwent magnetic resonance imaging of the liver. Hepatic focal lesions were classified according to LI-RADS-v2018, and ADC of hepatic lesions was calculated by 2 independent blinded reviewers.

Results: The mean ADC of LR-1 and LR-2 were $2.11 \pm 0.47$ and $2.08 \pm 0.47 \times 10^{-3} \mathrm{~mm}^{2} / \mathrm{s}$, LR-3 were $1.28 \pm 0.12$ and $1.36 \pm 0.16 \times 10^{-3} \mathrm{~mm}^{2} / \mathrm{s}$, LR-4, LR-5 and LR-TIV were $1.07 \pm 0.08$ and $1.08 \pm 0.12 \times 10^{-3} \mathrm{~mm}^{2} / \mathrm{s}$ and LR-M were 1.02 \pm 0.09 and $1.00 \pm 0.09 \times 10^{-3} \mathrm{~mm}^{2} / \mathrm{s}$ by both observers, respectively. There was excellent agreement of both readings for LR-1 and LR-2 $(r=0.988)$, LR-3 $(r=0.965)$, LR-4, LR-5 and LR-TIV $(r=0.889)$ and LR-M $(r=0.883)$. There was excellent correlation between ADC and LI-RADS-v2018 $(r=-0.849$ and -0.846$)$. The cut-off ADC used to differentiate LR-3 from LR-4, LR-5, and LR-TIV were $\leq 1.21$ and $\leq 1.23 \times 10^{-3} \mathrm{~mm}^{2} / \mathrm{s}$ with AUC of 0.948 and 0.926 .

Conclusions: Inclusion of ADC to LI-RADS-v2018 improves differentiation variable LI-RADS categories and can helps in the prediction of HCC.

Key words: liver cancer, hepatocellular carcinoma, abdominal MRI scan, diagnostic imaging.

\section{Introduction}

Hepatocellular carcinoma (HCC) is the most common primary hepatic malignancy and the second leading cause of cancer-related mortality in the world; thus, early diagnosis of HCC is vital for proper management [1-4]. Recently, contrast-enhanced computed tomography (CT) or magnetic resonance imaging (MRI) has become routinely performed for the diagnosis of HCC and has replaced biopsy preceding treatment for most of the patients [1,2]. Also, contrast-enhanced CT and/or MRI plays a critical role in the differentiation between HCC and other hepatic malignancies [5-7]. The Liver Imaging Reporting and Data System (LI-RADS) is a radiology-driven and multidisciplinary cooperative categorization system planned for standardizing liver imaging in patients at increased risk for HCC [8-12]. LI-RADS was originally released by the American College of Radiology (ACR) in 2011, and was then updated 4 times in 2013, 2014, 2017, and 2018. According to the previously released LI-RADS versions, established HCC imaging features include arterial phase hyperenhancement, washout appearance, and capsule appearance; also, the combination of both size and growth rate with these features is essential for the imaging algo-

Correspondence address:

Gehad Saleh, MD, Department of Diagnostic Radiology, Mansoura Faculty of Medicine, Elgomhoria St., Mansoura, 35512, Egypt,

e-mail: gehadsaleh@mans.edu.eg

Authors' contribution:

A Study design · B Data collection · C Statistical analysis · D Data interpretation · E Manuscript preparation · F Literature search · G Funds collection 
rithm [13-15]. The LI-RADS system meets the necessity to perform an exact diagnosis of HCC; actually, it is clinically important to distinguish between HCC and other hepatic malignancies because the management changes substantially. There are multiple ancillary features favouring malignancy, which could be used to upgrade the LI-RADS categories such as mild to moderate hyperintensity on T2-weighted imaging and restricted diffusion [14-18].

Diffusion-weighted imaging (DWI) is a functional technique used to assess tissue cellularity based on the restriction of water molecule motion. The analysis of DW images can be done qualitatively and quantitatively, through the apparent diffusion coefficient (ADC) map [16-18]. The role of DWI has been established in diagnosis, prognosis, and follow-up after treatment of malignancy of the brain, breast, chest, and head and neck regions. Several studies have discussed the role of DWI in the detection of hepatic malignancies and the differentiation of HCC from dysplastic nodules [19-22]. Multiple studies have discussed the role of incorporation of the ADC value into the Prostate Imaging Reporting and Data System (PI-RADS) [23-26]. Few studies have discussed the value of DWI and ADC in the characterization of hepatic focal lesions in correlation to LI-RADS [27-30]. The uniqueness of this study is the trial to directly compare the diagnostic accuracy and inter-observer agreement between ADC values of variable hepatic observations according to the recently released LI-RADS-v2018 and to assess prospective inclusion of ADC with LI-RADS-v2018 for the prediction of HCC.

The aim of the study was to assess the value of inclusion of the ADC value in LI-RADS-v2018 for better differentiation variable LI-RADS and prediction of HCC.

\section{Material and methods}

\section{Patients}

This study was approved by the institutional review board, and informed consent was waived because this is a retrospective study. The inclusion criteria were untreated patients at risk for HCC with hepatic focal lesion, including patients with cirrhosis (92 patients), patients with chronic hepatitis B infection (14 patients), and patients with prior HCC (2 patients). We excluded 5 patients from the study due to diffusion image quality degradation caused by respiratory motion artifacts. Finally, 137 hepatic focal lesions in 108 patients ( 75 male and 33 female, age range $40-66$ years) were included in the study. All patients underwent contrastenhanced dynamic MR examination and DWI of the liver in the period between February 2019 and March 2020.

\section{Magnetic resonance imaging technique}

All patients included in this study underwent MR imaging using a 1.5-T MR imaging scanner (Philips Inge- nia). Patients fasted for 4-6 hours and were instructed to avoid deep breathing during the examination. Precontrast T1-weighted imaging and T2-weighted imaging were acquired. Multiphasic post-contrast T1-weighted GRE sequence after injection of contrast medium (gadolinium chelate) (Gd-DTPA) at a dose of $0.1 \mathrm{mmol} / \mathrm{kg}$ was done with the following acquisition parameters: TR/TE 3.3-4.5/1.4-1.9 ms, flip angle $10^{\circ}$, number of excitations (NEX) 2, matrix size $172 \times 135$, field of view $300-400 \mathrm{~mm}$, and slice thickness of 3-5 mm. DWI was performed before contrast study using fat-suppressed single-shot echo-planar sequence with $b$ values $(b=0,100,600$, and $1000 \mathrm{~s} / \mathrm{mm}^{2}$ ). Diffusion imaging acquisition parameters were as follows: $\mathrm{TR} / \mathrm{TE}=1900-70 \mathrm{~ms}, \mathrm{NEX}=3$, matrix $=$ $124 \times 120$, slice thickness $=5 \mathrm{~mm}$, slice gap $=1-2 \mathrm{~mm}$, and scan time $=70 \mathrm{sec}$.

\section{Image analysis}

Using a workstation (Phillips Advantage windows workstation) all MR images were interpreted by 2 independent radiologists (AA and AG) with 25 and 10 years of experience in liver imaging, respectively. Both reviewers independently reviewed the major imaging features on CEMRI images, DWIs, and liver segments for each lesion, then the final diagnosis was arrived at for each observation according to LI-RADS-v2018 four major imaging features counting non-rim arterial phase hyperenhancement (APHE), non-peripheral washout appearance, capsule appearance, and focal lesion size. Finally, focal lesions were classified into 7 groups: LR-1 (definitively benign), LR-2 (probably benign), LR-3 (indeterminate), LR-4 (probably HCC), and LR-5 (definitively HCC), LR-M (probably or definitely malignant but not necessarily HCC), and LRTIV (tumour in vein) [31]. We considered LI-RADS-v2018 major imaging features on dynamic MRI as the standard of reference for 106 (77\%) typical hepatic observations (26 LR-1, 21 LR-2, 5 LR-3, 8 LR-4, 25 LR-5, 16 LR-M, and 5 LR-TIV). While the residual 31 (23\%) atypical hepatic observations the following approaches were considered for proper diagnosis; for 3 cases LR-3, dynamic MRI with diffusion was repeated within 3-6 months, 6 cases of LR-4 underwent biopsy before locoregional treatment and liver transplantation, 7 cases of LR- 5 also underwent biopsy before liver transplantation, for 11 cases of LR-M 3 of them underwent segmentectomy and 8 cases underwent biopsy, and finally 4 cases of LR-TIV underwent biopsy.

Restricted diffusion was considered as hyperintensity signal on DWI at high $b$ values of 600 and $1000 \mathrm{~mm}^{2} / \mathrm{s}$ and iso- or hypointensity signal on the corresponding ADC map relative to neighbouring liver parenchyma. Both reviewers measured ADC values separately by manually applying the region of interest (ROI) encompassing the homogenous solid part of the hepatic focal lesion and did not include adjacent hepatic parenchyma. The ADC values were measured 3 times, and the measurements were 
averaged. In 13 patients with multiple observations, after exclusion of observations less than $10 \mathrm{~mm}$ (7 hepatic observations), each one was determined by its location in the liver (according to the hepatic segment) and was allocated an identifying number (e.g. Observation \#1) to avoid overlap between the 2 observers. They were then analysed separately, the average ADC value was calculated for each observation, and finally the patient was classified according to the highest LI-RADS category.

\section{Statistical analysis}

The statistical analysis of data was done using the SPSS software (Statistical Package for the Social Sciences version 22). The mean and standard deviation of the ADC value was calculated. Two-tailed Student's test was used for comparison of parametric data of ADC values. The $\kappa$ values were interpreted as follows: $\kappa$ values between 0.61 and 0.80 represented good; and $\kappa$ values between 0.81 and 1.00 represented excellent. A p-value of less than 0.05 indicated a statistically significant difference. The receiver operating characteristic curves of the ADC of different categories of LI-RADS with a calculation of the area under the curve (AUC) was done. The weighted kappa test ( $\kappa$ ) was done to estimate the level of agreement for ADC value by both reviewers. Pearson's correlation test was done to correlate ADC values with LI-RADS-v2018.

\section{Results}

Table 1 shows the number of hepatic observations within each LI-RADS category. However, in this study we focused on evaluation of both DWI and ADC measurements in the assessment of different LI-RADs categories. Table 2 shows the mean and standard deviations of ADC of LI-RADS-v2018 subtypes. The mean ADC of both reviewers ( $b$ value $600 \mathrm{~mm}^{2} / \mathrm{s}$ ) of LR-1 and LR-2 were 2.11 \pm 0.47 and $2.08 \pm 0.47 \times 10^{-3} \mathrm{~mm}^{2} / \mathrm{s}$, LR-3 (Figures 1-3) were $1.28 \pm 0.12$ and $1.36 \pm 0.16 \times 10^{-3} \mathrm{~mm}^{2} / \mathrm{s}$, LR-4, LR-5 (Figures 4-6) and LR-TIV were $1.07 \pm 0.08$ and $1.08 \pm 0.12$ $\times 10^{-3} \mathrm{~mm}^{2} / \mathrm{s}$ and LR-M (Figures 7-9) were $1.02 \pm 0.09$ and $1.00 \pm 0.09 \times 10^{-3} \mathrm{~mm}^{2} / \mathrm{s}$. The ADC of LR-1, LR-2 was significantly different than that of LR-3, LR-4, LR-5 with a $p$-value of 0.001 for both reviewers.

Table 3 shows the ROC results of both readings of ADC values used for differentiation of LI-RADS subtypes.
The cut-off ADC values used to differentiate LR-1, LR-2 from LR-3 by both observers were $\leq 1.442$ and $\leq 1.498$ $\times 10^{-3} \mathrm{~mm}^{2} / \mathrm{s}$ with AUCs of 0.97 and 0.92 , respectively (Figure 10). The cut-off ADC values used to differentiate LR-3 from LR-4, LR-5, and LR-TIV by both observers were $\leq 1.214$ and $\leq 1.238 \times 10^{-3} \mathrm{~mm}^{2} / \mathrm{s}$ with AUCs of 0.948 and 0.926 , respectively (Figure 11). The cut-off ADC values used to differentiate LR-M from LR-4, LR-5, and LR-TIV by both observers were $\leq 1.026$ and $\leq 1.001 \times 10^{-3} \mathrm{~mm}^{2} / \mathrm{s}$ with AUCs of 0.694 and 0.732 , respectively (Figure 12 ).

There was statistically significant excellent agreement between both readings of ADC values for LR-1 and LR-2 $(\kappa=0.988, p=0.001)$, LR-4, LR-5 and LR-TIV $(\kappa=0.889, p=0.001)$, and LR-M $(\kappa=0.883, p=0.001)$. There was statistically significant excellent negative correlation of ADC and LI-RADS-v2018 ( $r=-0.849$ and -0.84 , $p=0.001$ ) by both observers, respectively.

\section{Discussion}

In the current study, the ADC of LR-1,2 was significantly higher $(p=0.001)$ than that of LR-3, LR-4, LR-5 for both reviewers. There is a significant excellent agreement of ADC values for the evaluation of LI-RADS-v2018 by both reviewers, and there was a significant-excellent correlation between both readings of ADC and LI-RADS-v2018.

Precise differentiation between HCC and other malignant hepatic focal lesions is crucial because the treatment and prognosis differ significantly. Multiple previous updates and studies have been made to improve the LI-RADS classification system since its development, for a confident diagnosis of HCC [13-15]. In this study, not only dynamic contrast-

Table 1. Number of observations within each LI-RADS category by both observers

\begin{tabular}{|l|c|c|} 
& Observer 1 & Observer 2 \\
\hline LR-1 & 26 & 26 \\
\hline LR-2 & 21 & 21 \\
\hline LR-3 & 8 & 6 \\
\hline LR-4 & 14 & 18 \\
\hline LR-5 & 33 & 32 \\
\hline LR-M & 27 & 26 \\
\hline LR-TIV & 8 & 8 \\
\hline
\end{tabular}

Table 2. ADC (x 10-3 mm2/s) of LI-RADS v2018 of both reviewers with inter-observer agreement

\begin{tabular}{|l|c|c|c|} 
& Observer 1 & Observer 2 & ICC \\
\hline ADC LR-1,2 & $2.11 \pm 0.47(1.31-3.04)$ & $2.08 \pm 0.47(1.33-3.12)$ & $r=0.988, p=0.001$ \\
\hline ADC LR-3 & $1.28 \pm 0.12(1.11-1.44)$ & $1.36 \pm 0.16(1.20-1.51)$ & $r=0.965, p=0.035$ \\
\hline ADC LR-4,5, TIV & $1.07 \pm 0.08(0.94-1.29$ & $1.08 \pm 0.12(0.90-1.41)$ & $r=0.889, p=0.001$ \\
\hline ADC LR-M & $1.02 \pm 0.09(0.94-1.26)$ & $1.00 \pm 0.09(0.90-1.31)$ & $r=0.883, p=0.001$ \\
\hline
\end{tabular}

$A D C$ - apparent diffusion coefficient 


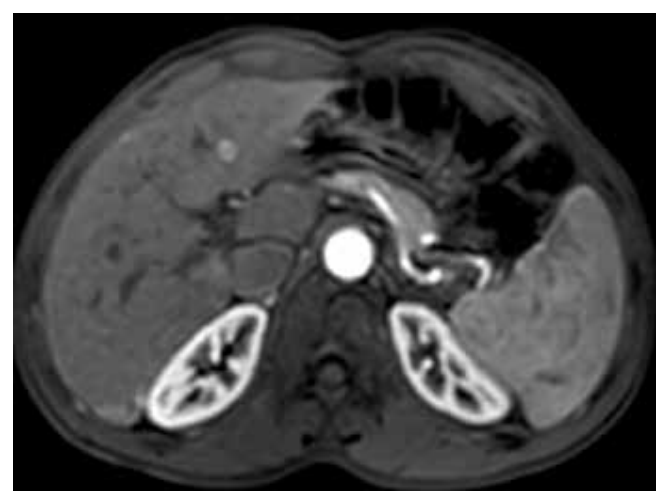

Figure 1. Axial arterial phase image shows a small focal lesion with hyperenhancement

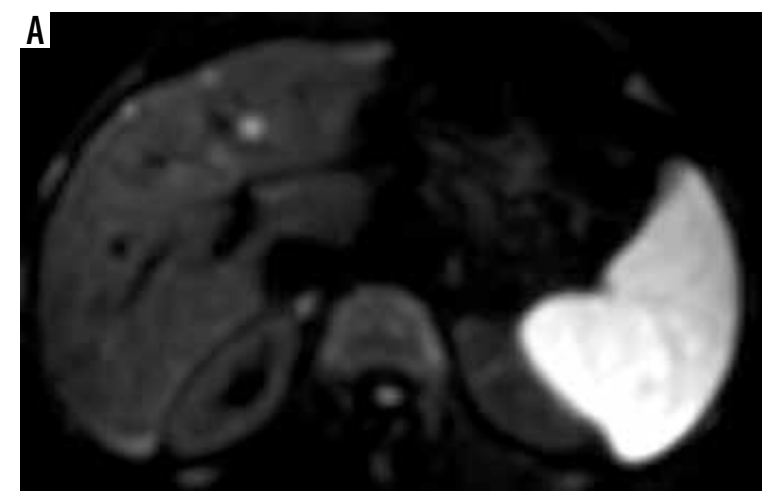

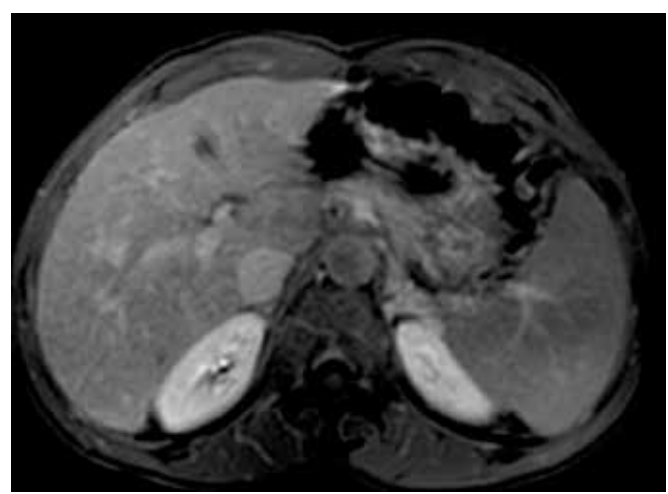

Figure 2. Delayed phase image shows no washout or enhancing capsule appearance

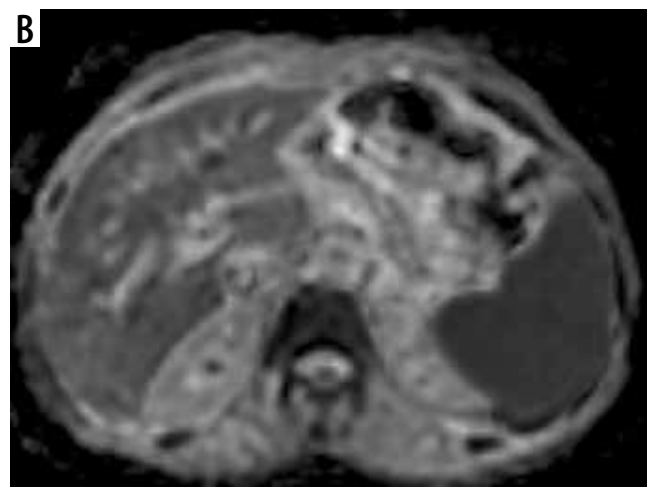

Figure 3. DWI ( $b$ value $\left.=600 \mathrm{~mm}^{2} / \mathrm{s}\right)$ and $A D C$ map shows restricted diffusion with $A D C$ value of hepatic focal lesion $\left(1.31\right.$ and $\left.1.38 \times 10^{-3} \mathrm{~mm}^{2} / \mathrm{s}\right)$ by both reviewers, respectively

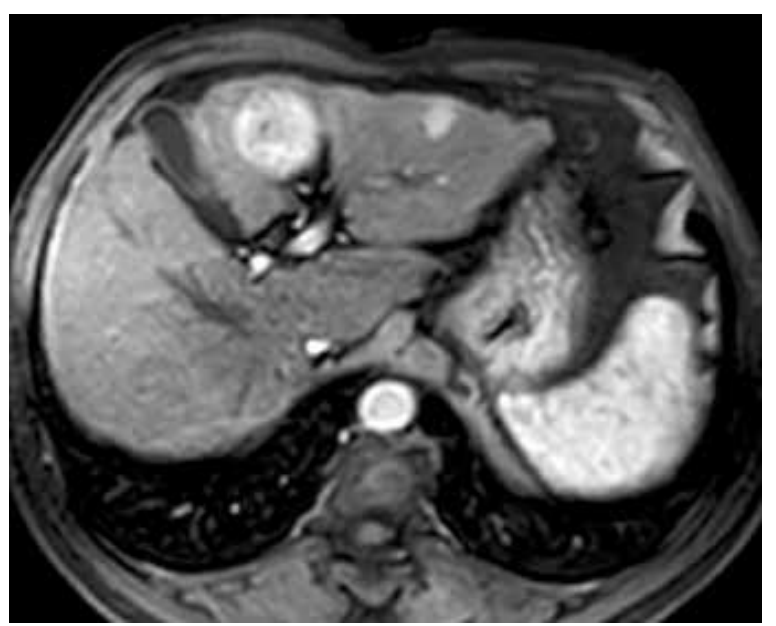

Figure 4. Axial arterial phase image shows a large focal lesion in segment IV and another smaller lesion in segment III with similar arterial phase hyperenhancement

enhanced MRI but also DWI and quantitative ADC analysis were used to differentiate between different hepatic focal lesions according to the recently released LI-RADS-v2018.

We performed a recent study concerned with LI-RADSv2018 major imaging features and revealed excellent inter-observer agreement for LR-1, LR- 2, LR-5, LR-M, and LR-TIV with good agreement for LR-3 and LR-4 and excellent inter-observer agreement for the major imaging features [31]. So, in the current study we focused on both DWI and ADC analysis, the ADC value used

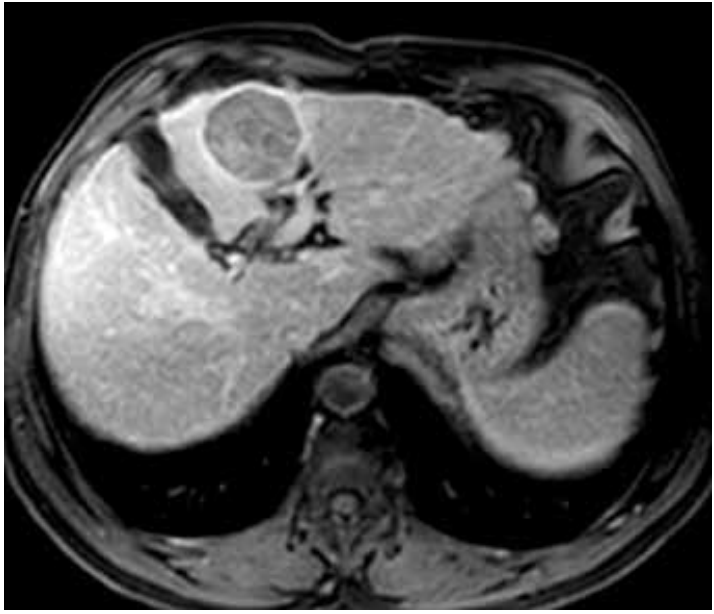

Figure 5. Delayed phase image shows washout and enhancing capsule appearance

to differentiate LR-3 from LR-1, LR-2 with AUC of 0.97 and 0.92 , respectively, and from LR-4, LR-5, and LR-TIV with 0.948 and 0.926 by both observers, respectively.

A recent study showed that for prostate lesions with a PI-RADS score $\geq 3$, ADC values calculated prospectively can help discriminate clinically insignificant from clinically significant prostate cancer, allowing pre-biopsy and pretreatment risk stratification [23]. A retrospective study was performed to define the diagnostic accuracy of ADC values in association with PI-RADS-v2 for 
Table 3. ROC of ADC of LI-RADS-v2018 categories with AUC, accuracy, sensitivity, and specificity of both reviewers

\begin{tabular}{|c|c|c|c|c|c|}
\hline & Cut-off & AUC & Sensitivity & Specificity & Accuracy \\
\hline \multicolumn{6}{|c|}{ LR-3 versus LR-1,2 } \\
\hline $1^{\text {st }}$ reviewer & $\leq 1.44$ & 0.970 & 83.3 & 93.2 & 92.0 \\
\hline $2^{\text {nd }}$ reviewer & $\leq 1.49$ & 0.920 & 75.0 & 84.1 & 83.3 \\
\hline \multicolumn{6}{|c|}{ LR-3 versus LR 4, 5, TIV } \\
\hline $1^{\text {st }}$ reviewer & $\leq 1.21$ & 0.948 & 95.6 & 83.3 & 94.1 \\
\hline $2^{\text {nd }}$ reviewer & $\leq 1.23$ & 0.926 & 91.5 & 75.0 & 90.2 \\
\hline \multicolumn{6}{|c|}{ LR- M versus L4,5, TIV } \\
\hline $1^{\text {st }}$ reviewer & $\leq 1.02$ & 0.694 & 66.7 & 55.6 & 59.4 \\
\hline $2^{\text {nd }}$ reviewer & $\leq 1.00$ & 0.723 & 70.8 & 63.8 & 66.2 \\
\hline
\end{tabular}

$A D C$ - apparent diffusion coefficient, $A U C$ - area under the curve
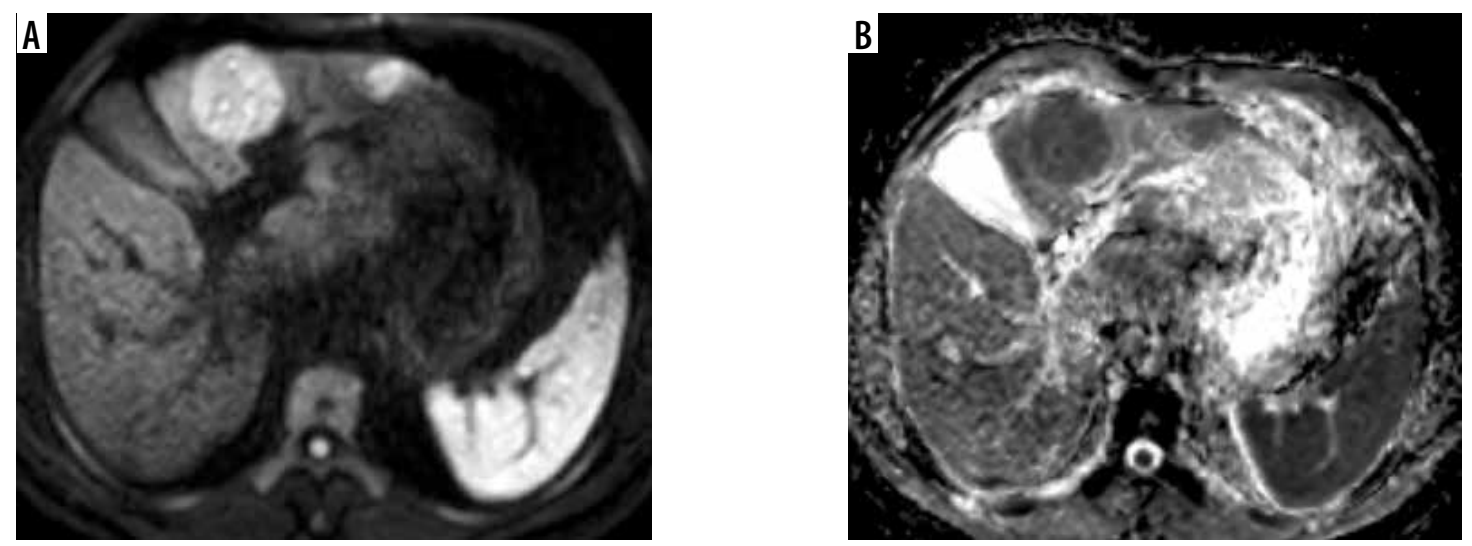

Figure 6. DWI (b value $\left.=600 \mathrm{~mm}^{2} / \mathrm{s}\right)$ and $A D C$ map show diffusion restriction of both lesions with $A D C$ values of the large lesion $(0.98$ and $1.06 \times 10-3$ $\left.\mathrm{mm}^{2} / \mathrm{s}\right)$ and for the smaller lesion ( 1.11 and $\left.1.04 \times 10-3 \mathrm{~mm}^{2} / \mathrm{s}\right)$ by both reviewers, respectively

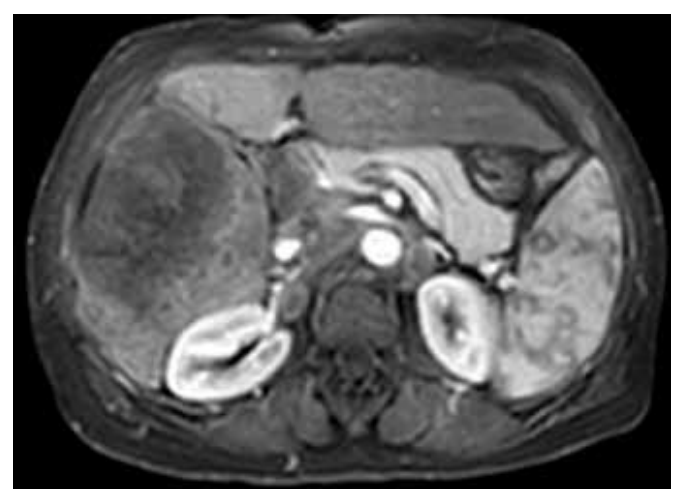

Figure 7. Axial arterial phase image shows rim arterial phase hyperenhancement

the diagnosis of clinically significant prostate cancer in comparison to PI-RADS-v2 alone; the results revealed that the maximum benefit of incorporating absolute ADC values was in lesions with a PI-RADS-v2 score of 4 [24].

Prior studies were designed to assess the value of DWI and measured ADC values in a selected group of hepatic focal lesions, mainly concerned with differentiating between benign and malignant lesions. A prospective recent study evaluated the value of DWI in improving

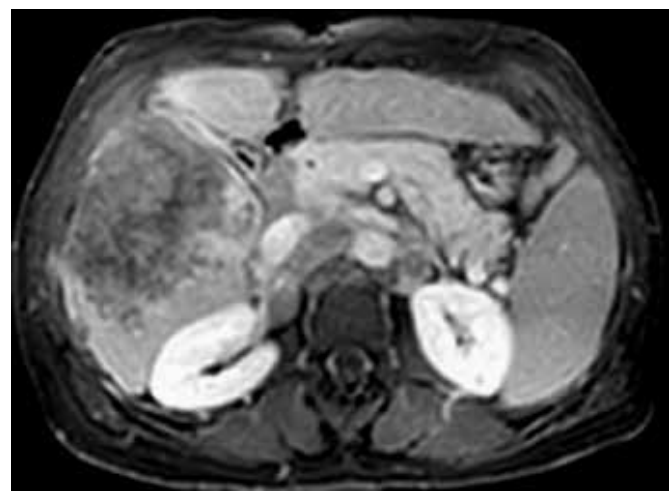

Figure 8. Delayed phase image shows progressive enhancement

the sensitivity of LI-RADS classification of small hepatic lesions ( $\leq 20 \mathrm{~mm}$ ), which were formerly characterized as LI-RADS grade 3-5 on dynamic contrast-enhanced CT [28], unlike our study, in which we included all variable LI-RADS v2018 categories. One retrospective study evaluated the performance DWI and T2-weighted imaging in the detection of HCC in reference to the LI-RADS version 2014 with only LI-RADS grade 3-5 lesions [29], while in our study we included all LI-RADS categories and LI-RADS major imaging features as the reference 

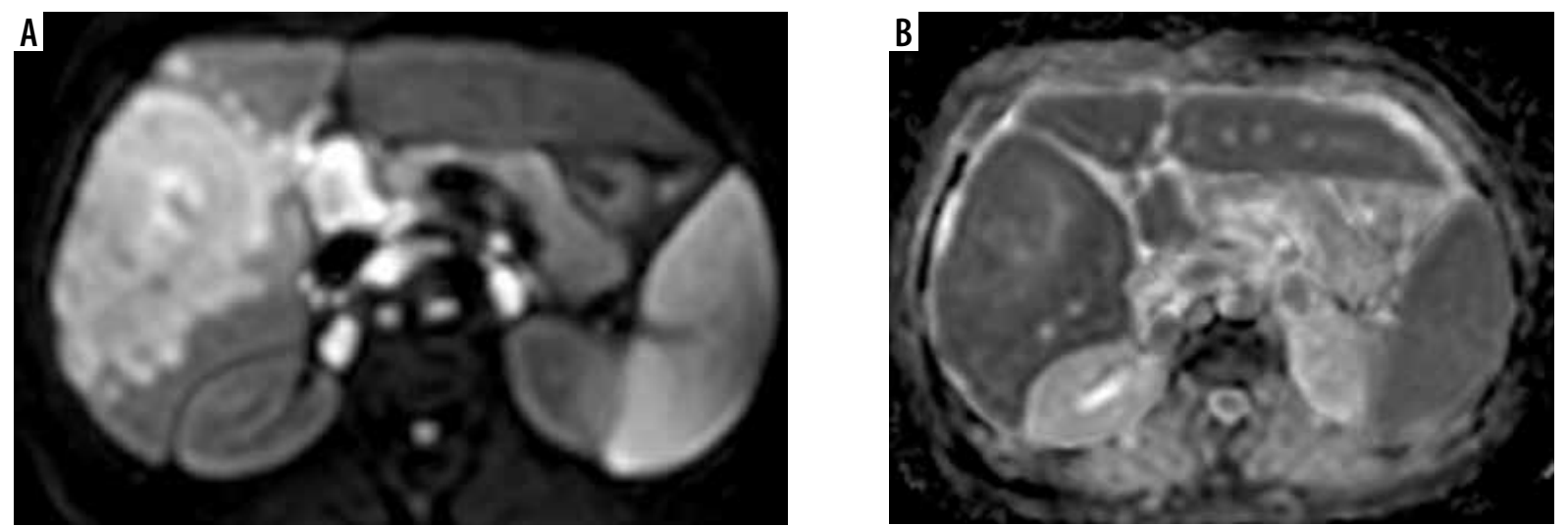

Figure 9. DWI ( $b$ value $\left.=600 \mathrm{~mm}^{2} / \mathrm{s}\right)$ and $A D C$ map show diffusion restriction with $A D C$ values $\left(0.95\right.$ and $\left.0.97 \times 10^{-3} \mathrm{~mm}^{2} / \mathrm{s}\right)$ by both reviewers, respectively

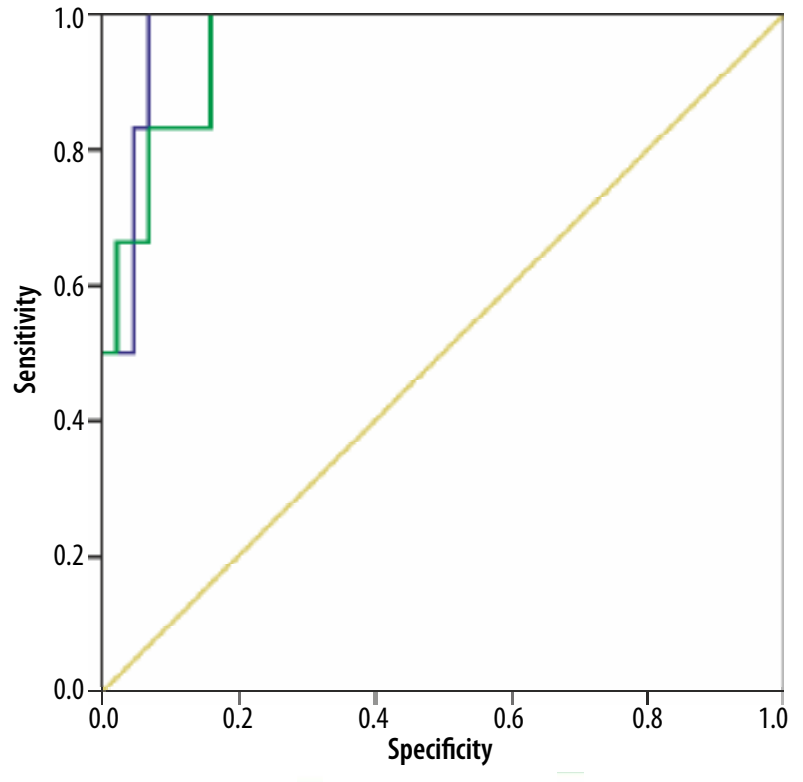

$-\mathrm{ADC}$ (first observer) $-\mathrm{ADC}$ (second observer) - Reference line

Figure 10. Cut-off ADC values used to differentiate LR-1, LR-2 from LR-3 by both observers were $\leq 1.442$ and $\leq 1.498 \times 10-3 \mathrm{~mm}^{2} / \mathrm{s}$ with AUCs of 0.97 and 0.92 , respectively

standard. A recent study used ADC values to differentiate between haemangioma and HCC, where ADC values of haemangiomas were significantly higher than those of HCC [30]. Another recent study stated that quantitative ADC histogram analysis increase the accuracy for the diagnosis of HCC compared to the other primary liver cancers, and the combination of quantitative ADC measurement and LI-RADS improve this distinction [27].

In this study, the inter-observer agreement of the ADC value of different categories of LI-RADS-v2018 is excellent. One study reported that the inter-reader agreement of the LI-RADS scores between combined technique and dynamic contrast MR imaging is good $(\kappa=0.765)$ [28]. Another study added that there is an excellent inter-observer agreement of both reviewers for LI-RADS-v2018 $(\kappa=0.887, p=0.001)$ with $90.76 \%$ agreement [31].

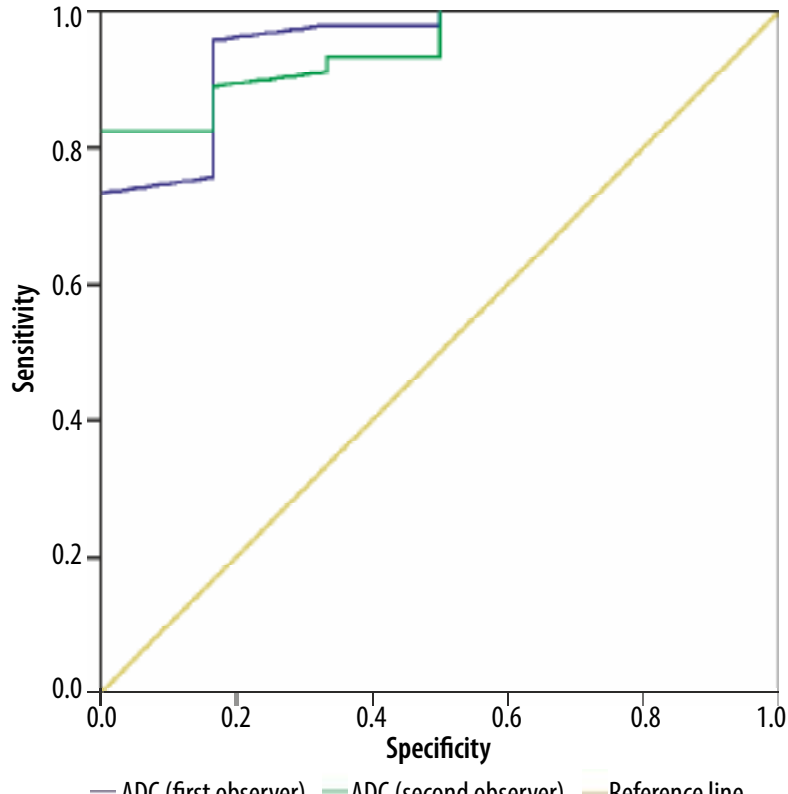

Figure 11. Cut-off $A D C$ value used to differentiate $L R-3$ from $L R-4, L R-5$, and LR-TIV by both observers were $\leq 1.214$ and $\leq 1.238 \times 10-3 \mathrm{~mm} 2 / \mathrm{s}$ with AUCs of 0.948 and $0.926 \times 10-3 \mathrm{~mm} 2 / \mathrm{s}$, respectively

In our study, there is a statistically significant-excellent negative correlation between ADC and LI-ARDS classification for both reviewers. One study reported that ADC and normalized ADC inversely correlates with PI-RADSv2 and International Society of Urological Pathology Systems categories [26]. Another study added that ADC values and categories help to diagnose clinically significant prostate cancer when lesions are assigned a PI-RADS v2 score of 4 [24].

There were a few limitations to our study. First, this is a retrospective design of the study with a small number of patients. Further prospective studies of a large number of patients are recommended. Second, this study included untreated hepatic focal lesions; further studies including hepatic focal lesions undergoing locoregional treatment [32] are recommended. Third, this study used only DWI and ADC values for evaluating 
hepatic focal lesions; further studies using advanced MR techniques such as diffusion tensor imaging, arterial spin labelling, perfusion MR imaging, and MR spectroscopy with a higher 3-Tesla scanner [33-49] with LI-RADS-v2018 are recommended. Fourth, image analysis was performed by ROI localization; further studies with applied advanced post-processing such as machine learning and histogram analysis are advised for standard ROI size $[50,51]$.

\section{Conclusions}

The inclusion of ADC values of hepatic focal lesions to the standard interpretation of LI-RADS-v2018 can improve accuracy, sensitivity, and specificity. Also, it may help in the early prediction of HCC. ADC measurement is a promising ancillary feature, which should be routinely performed for better clarification of different LI-RADS categories.

\section{Conflicts of interest}

The authors report no conflict of interest.

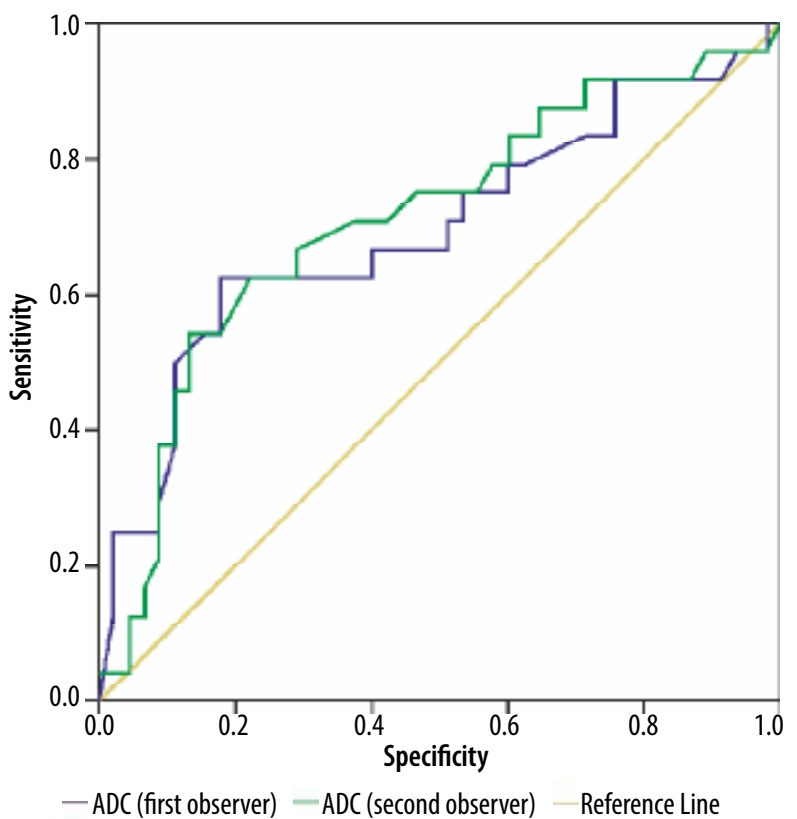

Figure 12. Cut-off $A D C$ value used to differentiate $L R-M$ from $L R-4, L R-5$, and LR-TIV by both observers were $\leq 1.026$ and $\leq 1.001$ with AUCs of 0.694 and 0.732 , respectively

\section{References}

1. Villanueva A. Hepatocellular carcinoma. N Engl J Med 2019; 380: 1450-1462.

2. Harris PS, Hansen RM, Gray ME, et al. Hepatocellular carcinoma surveillance: an evidence-based approach. World J Gastroenterol 2019; 25: 1550-1559.

3. Desai A, Sandhu S, Lai JP, et al. Hepatocellular carcinoma in non-cirrhotic liver: a comprehensive review. World J Hepatol 2019; 11: 1-18.

4. Elshaarawy O, Gomaa A, Omar H, et al. Intermediate stage hepatocellular carcinoma: a summary review. J Hepatocell Carcinoma 2019; 6: 105-117.

5. Ronot M, Purcell Y, Vilgrain V. Hepatocellular carcinoma: current imaging modalities for diagnosis and prognosis. Dig Dis Sci 2019; 64 934-950.

6. Fetzer DT, Rodgers SK, Seow JH, et al. Ultrasound evaluation in patients at risk for hepatocellular carcinoma. Radiol Clin North Am 2019; 57: 563-583.

7. An C, Kim MJ. Imaging features related with prognosis of hepatocellular carcinoma. Abdom Radiol (NY) 2019; 44: 509-516.

8. Ren AH, Zhao PF, Yang DW, et al. Diagnostic performance of MR for hepatocellular carcinoma based on LI-RADS v2018, compared with v2017. J Magn Reson Imaging 2019; 50: 746-55.

9. Kielar AZ, Chernyak V, Bashir MR, et al. An update for LI-RADS: version 2018. Why so soon after version 2017? J Magn Reson Imaging 2019; 50: 1990-1991.

10. Kim YY, Kim MJ, Kim EH, et al. Hepatocellular carcinoma versus other hepatic malignancy in cirrhosis: performance of LI-RADS version 2018. Radiology 2019; 291: 72-80.

11. Tang A, Singal AG, Mitchell DG, et al. Introduction to the Liver Imaging Reporting and Data System (LI-RADS) for hepatocellular carcinoma. Clin Gastroenterol Hepatol 2019; 17: 1228-1238.
12. van der Pol CB, Lim CS, Sirlin CB, et al. Accuracy of the liver imaging reporting and data system in computed tomography and magnetic resonance image analysis of hepatocellular carcinoma or overall malignancy - a systematic review. Gastroenterology 2019; 156: 976-986.

13. Elsayes KM, Kielar AZ, Elmohr MM, et al. White paper of the Society of Abdominal Radiology hepatocellular carcinoma diagnosis disease-focused panel on LI-RADS v2018 for CT and MRI. Abdom Radiol 2018; 43: 2625-2642.

14. Abdel Razek AAK, El-Serougy LG, Saleh GA, et al. Liver imaging reporting and data system version 2018: what radiologists need to know. J Comput Assist Tomogr 2020; 44: 168-177.

15. Chernyak V, Fowler KJ, Kamaya A, et al. Liver Imaging Reporting and Data System (LI-RADS) version 2018: imaging of hepatocellular carcinoma in at-risk patients. Radiology 2018; 289: 816-830.

16. Abdel Razek AA, Soliman N, Elashery R. Apparent diffusion coefficient values of mediastinal masses in children. Eur J Radiol 2012; 81: 1311-1314.

17. Razek AAKA, Massoud SMA, Azziz MRA, et al. Prediction of esophageal varices in cirrhotic patients with apparent diffusion coefficient of the spleen. Abdom Imaging 2015; 40: 1465-1469.

18. Razek AAKA, Abdalla A, Omran E, et al. Diagnosis and quantification of hepatic fibrosis in children with diffusion weighted MR imaging. Eur J Radiol 2011; 78: 129-134.

19. Wei Y, Gao F, Wang M, et al. Intravoxel incoherent motion diffusion-weighted imaging for assessment of histologic grade of hepatocellular carcinoma: comparison of three methods for positioning region of interest. Eur Radiol 2019; 29: 535-544.

20. Taron J, Johannink J, Bitzer M, et al. Added value of diffusion-weighted imaging in hepatic tumors and its impact on patient management. Cancer Imaging 2018; 18: 10. 
21. Ogihara Y, Kitazume Y, Iwasa Y, et al. Prediction of histological grade of hepatocellular carcinoma using quantitative diffusion-weighted MRI: a retrospective multivendor study. Br J Radiol 2018; 91: 20170728.

22. Budjan J, Sauter EA, Zoellner FG, et al. Diffusion kurtosis imaging of the liver at 3 Tesla: in vivo comparison to standard diffusion-weighted imaging. Acta Radiol 2018; 59: 18-25.

23. Costa DN, Xi Y, Aziz M, et al. Prospective inclusion of apparent diffusion coefficients in multiparametric prostate mri structured reports: discrimination of clinically insignificant and significant cancers. AJR Am J Roentgenol 2019; 212: 109-116.

24. Jordan EJ, Fiske C, Zagoria R, et al. PI-RADS v2 and ADC values: is there room for improvement? Abdom Radiol 2018; 43: 3109-3116.

25. Polanec SH, Helbich TH, Bickel H, et al. Quantitative apparent diffusion coefficient derived from diffusion-weighted imaging has the potential to avoid unnecessary MRI-guided biopsies of mpMRI-detected PI-RADS 4 and 5 lesions. Invest Radiol 2018; 53: 736-741.

26. Gaur S, Harmon S, Rosenblum L, et al. Can apparent diffusion coefficient values assist PI-RADS version 2 DWI scoring? A correlation study using the PI-RADSv2 and International Society of Urological Pathology systems. AJR Am J Roentgenol 2018; 211: W33-41.

27. Lewis S, Peti S, Hectors SJ, et al. Volumetric quantitative histogram analysis using diffusion-weighted magnetic resonance imaging to differentiate HCC from other primary liver cancers. Abdom Radiol 2019; 44: 912-922.

28. Basha MAA, Refaat R, Mohammad FF, et al. The utility of diffusion-weighted imaging in improving the sensitivity of LI-RADS classification of small hepatic observations suspected of malignancy. Abdom Radiol 2019; 44: 1773-1784.

29. Hicks RM, Yee J, Ohliger MA, et al. Comparison of diffusion-weighted imaging and T2-weighted single shot fast spin-echo: Implications for LI-RADS characterization of hepatocellular carcinoma. Magn Reson Imaging 2016; 34: 915-921.

30. Nam SJ, Yu JS, Cho ES, et al. High-flow haemangiomas versus hypervascular hepatocellular carcinoma showing "pseudo-washout" on gadoxetic acid-enhanced hepatic MRI: value of diffusion-weighted imaging in the differential diagnosis of small lesions. Clin Radiol 2017; 72: 247-254.

31. Abdel Razek AAK, El-Serougy LG, Saleh GA, et al. Interobserver agreement of magnetic resonance imaging of liver imaging reporting and data system version 2018. J Comput Assist Tomogr 2020; 44: 118-123.

32. Abdel Razek AAK, El-Serougy LG, Saleh GA, et al. Reproducibility of LI-RADS treatment response algorithm for hepatocellular carcinoma after locoregional therapy. Diagn Interv Imaging 2020; 101: 547-553.

33. Razek AAKA. Diffusion tensor imaging in differentiation of residual head and neck squamous cell carcinoma from post-radiation changes. Magn Reson Imaging 2018; 54: 84-89.

34. Khalek Abdel Razek AA. Characterization of salivary gland tumours with diffusion tensor imaging. Dentomaxillofac Radiol 2018; 47: 20170343.

35. El-Serougy L, Abdel Razek AA, Ezzat A, et al. Assessment of diffusion tensor imaging metrics in differentiating low-grade from highgrade gliomas. Neuroradiol J 2016; 29: 400-407.

36. Abdel Razek AAK, Talaat M, El-Serougy L, et al. Clinical applications of arterial spin labeling in brain tumors. J Comput Assist Tomogr 2019; 43: 525-532.
37. Abdel Razek AAK. Arterial spin labelling and diffusion-weighted magnetic resonance imaging in differentiation of recurrent head and neck cancer from post-radiation changes. J Laryngol Otol 2018; 132: 923-928.

38. Razek AAKA. Multi-parametric MR imaging using pseudo-continuous arterial-spin labeling and diffusion-weighted MR imaging in differentiating subtypes of parotid tumors. Magn Reson Imaging 2019; 63: 55-59.

39. Abdel Razek AA, Gaballa G, Ashamalla G, et al. Dynamic susceptibility contrast perfusion-weighted magnetic resonance imaging and diffusion-weighted magnetic resonance imaging in differentiating recurrent head and neck cancer from postradiation changes. J Comput Assist Tomogr 2015; 39: 849-854.

40. Razek AA, Nada N. Correlation of choline/creatine and apparent diffusion coefficient values with the prognostic parameters of head and neck squamous cell carcinoma. NMR Biomed 2016; 29: 483-489.

41. Surov A, Nagata S, Razek AA, et al. Comparison of ADC values in different malignancies of the skeletal musculature: a multicentric analysis. Skeletal Radiol 2015; 44: 995-1000.

42. Abdel Razek AA, Kamal E. Nasopharyngeal carcinoma: correlation of apparent diffusion coefficient value with prognostic parameters. Radiol Med 2013; 118: 534-539.

43. Abdel Razek AA, Elkhamary S, Al-Mesfer S, et al. Correlation of apparent diffusion coefficient at $3 \mathrm{~T}$ with prognostic parameters of retinoblastoma. AJNR Am J Neuroradiol 2012; 33: 944-948.

44. Razek AA, Lattif MA, Denewer A, et al. Assessment of axillary lymph nodes in patients with breast cancer with diffusion-weighted MR imaging in combination with routine and dynamic contrast MR imaging. Breast Cancer 2016; 23: 525-532.

45. Razek AA, Fathy A, Gawad TA. Correlation of apparent diffusion coefficient value with prognostic parameters of lung cancer. J Comput Assist Tomogr 2011; 35: 248-252.

46. Razek AAKA, El Badrawy MK, Alnaghy E. Interstitial lung fibrosis imaging reporting and data system: what radiologist wants to know? J Comput Assist Tomogr 2020; 44: 656-666.

47. Abdel Razek AAK, Abdelaziz TT. Neck imaging reporting and data system: what does radiologist want to know? J Comput Assist Tomogr 2020; 44: 527-532.

48. Abdel Razek AAK, Elrakhawy MM, Yossof MM, et al. Inter-observer agreement of the Coronary Artery Disease Reporting and Data System (CAD-RADS(TM)) in patients with stable chest pain. Pol J Radiol 2018; 83: e151-e159.

49. Abdel Razek AA, Ashmalla GA, Gaballa G, Nada N. Pilot study of Ultrasound Parotid Imaging Reporting and Data System (PIRADS): inter-observer agreement. Eur J Radiol 2015; 85: 2533-2538.

50. Razek AAKA. Editorial for "Preoperative MRI-based radiomic machine-learning nomogram may accurately distinguish between benign and malignant soft tissue lesions: a two-center study". J Magn Reson Imaging 2020; 52: 883-884.

51. Abdel Razek AAK. Routine and advanced diffusion imaging modules of the salivary glands. Neuroimaging Clin N Am 2018; 28: 245-254. 